\title{
HENOCH- SCHÖNLEIN PURPURA ASSOCIATED WITH MAJOR COMPLICATIONS
}

\author{
MOHAMMAD ZAHIDUL ISLAM
}

\begin{abstract}
Henoch Schönlein Purpura is a mild disease in children and usually self-limiting. Pulmonary hemorrhage is rare in prepubertal children. Our patient had rapidly progressive glomerulonephritis, pulmonary hemorrhage, nephrotic syndrome, hypertension, intermittent intussusception, and cerebral vasulitis with seizure. Combination of such severe complications in one patient over a short span of time is very rare. In our experience, we found that combination of iv methylprednisolone pulse therapy followed by oral prednisolone plus oral cyclophosphamide to be very effective.
\end{abstract}

Keyword: Henoch Schönlein Purpura, vasculitis, glomerulonephritis, pulmonary hemorrhage, intussusception

\section{Introduction}

Henoch Schönlein Purpura is a vasculitis syndrome involving small vessels and characterized by deposition of IgA1 in the renal mesangium and in the blood vessels. It can occur at any age but occurs mostly between the ages of 3 to 15 years, more commonly in boys. The onset of HSP maybe preceded by a upper respiratory tract infection. HSP is characterized by nonthrombocytopenic purpura, arthritis, arthralgia, abdominal pain, gastrointestinal hemorrhage and glomerulonephritis. The central nervous system is also occasionally affected, mostly with mild symptoms of headache and behavioral changes. There maybe evidence of cerebral vascultits. Intracerebral hemorrhage is very rare. Similarly, pulmonary hemorrhage is also a rare occurrence in HSP. It occurs mainly in adults and adolescents and is associated with significant morbidity and mortality. It has been reported only in few prepubertal children with slightly better outcome.

In most cases, HSP is a self-limiting disorder and tends to resolve within 4 weeks. However, recurrences are common. Most patients require only supportive care. Corticosteroids may be beneficial in patients with abdominal pain. The management for patients with more severe complications like pulmonary hemorrhage, glomerulonephritis remains unclear and controversial, but there is evidence that combination of high dose of corticosteroids and cytotoxic agents is beneficial in such cases. Although HSP can affect many systems in the body it is however not very common that a patient has combination of many severe complication at a time.

We describe a case, 10 years old Saudi girl presented with well defined Henoch Schönlein Purpura with multiple complications of it, yet treated successfully with corticosteroid and cyclophosphamide. She recovered completely from all complications and no recurrence was observed in follow up visits during the last 2 years.

\section{Case Report}

10-year-old Saudi girl was transferred from another hospital in Riyadh to our university hospital with presumptive diagnosis of Henoch Schönlein Purpura complicated by nephrotic syndrome, hypertension, acute pancreatitis and peritonitis. She was already on prednisolone, IV ceftriaxone and nifedipine. There was a history of upper respiratory infection 2-3 weeks before admission to that hospital. On receiving the patient in our renal unit, we found the patient to have hypertension with $\mathrm{BP}$ of $130 / 85 \mathrm{mmHg}$, bilateral pedal edema and ascitis. She had typical purpuric rash of HSP on the extensor surface of her legs and buttock. She complained of intermittent, on and off colicky abdominal pain and arthragia, mainly of bilateral knee joins. Her weight was 30 $\mathrm{kg}$, height $137 \mathrm{~cm}$, afebrile and other vitals were normal. Laboratory investigations showed serum

Correspondence: Mohammad Zahidul Islam, Consultant Pediatric Nephrologist, Department of Pediatrics (39), King Khalid University Hospital, PO Box: 11472, Riyadh, KSA, Email: Zahed1205@yahoo.com 
urea $1605 \mathrm{mmol} / \mathrm{l}$, creatinine $237 \mu \mathrm{mol} / \mathrm{l}$, Albumin 23 $\mathrm{g} / \mathrm{l}, \mathrm{Na}^{+} 137, \mathrm{~K}^{+} 3.9, \mathrm{Cl}^{-} 99, \mathrm{Ca}^{+2} 1.9$, Phosphate 2.0 $(\mathrm{mmol} / \mathrm{l})$, serum IgA $0.65 \mathrm{~g} / \mathrm{l}(\mathrm{N}), \mathrm{C}_{3}-1.28 \mathrm{~g} / \mathrm{l}(\mathrm{N}), \mathrm{C}_{4^{-}}$ $0.34 \mathrm{~g} / \mathrm{l}(\mathrm{N})$, ANA- negative, ANCA- negative, anti DS DNA antibody- negative. Serum Amylase 27 U/ $\mathrm{l}(\mathrm{N})$. Stool occult blood was negative. $\mathrm{Hb}: 8.7 \mathrm{~g} / \mathrm{l}$, WBC $12.5 \mathrm{X} 10^{9} / \mathrm{l}$, Platelet 21X10 $/ 1$, ESR $11 \mathrm{~mm}$ in $1^{\text {st }} \mathrm{hr}$. PT, APTT both were normal. Blood gas: $\mathrm{pH}$ : 7.46, $\mathrm{pCO}_{2} 30.5, \mathrm{pO}_{2} 44.1, \mathrm{HCO}_{3} 18 \mathrm{mmol} / \mathrm{l}$. Liver function test showed total bilirubin $7 \mu \mathrm{mol} / \mathrm{l}$ (direct $2 \mu \mathrm{mol} / \mathrm{l})$, ALP $93 \mathrm{u} / \mathrm{l}$, ALT $21 \mathrm{u} / \mathrm{l}$, AST $12 \mathrm{u} / \mathrm{l} \mathrm{GGT} 70$. Urinalysis: protein $4+$, blood $3+$, Urine protein/ creatinine ration: $0.8(\mathrm{~g} / \mathrm{mmol})$. There was $\mathrm{RBC}$, granular casts. Urine culture grew no organism. X ray Abdomen and ultrasonography were done twice, serum amylase was repeated and thus pancreatitis was virtually ruled out. Both kidneys were normal in shape and size but showed increased parenchymal echogenecity on both sides consistent with renal parenchymal disease. There was mild hepatomegaly with homogeneous parenchyma, spleen was normal, there was free fluid in abdomen and bilateral basal pleural effusion (which was seen in chest $\mathrm{X}$ ray also). A CT abdomen was done with contrast after giving i.v N-Acetyl cystein; it was suggestive of intramural hematoma in the region of terminal ileum, secondary to HSP vascultis. There was no evidence of pancreatitis, gallstones, cholecystitis or perforation. Skin biopsy revealed leukocytoclastic vascultis, IF was negative for IgA and $\mathrm{C}_{3}$. A percutaneous renal biopsy was also done on her left kidney, the result was still pending.

Our surgery colleagues were consulted for her colicky abdominal pain; they thought it was due to intermittent intussusception caused by intramural hematoma in the terminal ileum. No surgical intervention was necessary but she was kept on NPO (nothing by mouth). She was given given iv methyl prednisolone, instead of oral prednisolone, but in the same dose of $2 \mathrm{mg} / \mathrm{kg} /$ day (in divided doses). She was given iv albumin $20 \%$ with frusemide on few occasions. She was put on amlodipine for high blood pressure. Pleural effusion, ascitis and edema improved over the days, serum creatinine and urea level started to come down and she looked much better. However on day 5 , she had a brief right sided focal convulsion at night, she was however conscious. Her blood pressure at that time was $130 / 80 \mathrm{mmHg}$. All her electrolytes were normal except serum magnesium was slightly low $(0.6 \mathrm{mmol} / \mathrm{l})$. Urgent
CT followed by MRI brain was done, both suggested evidence of vasculitis on left frontal region but no active hemorrhage. She had another brief convulsion, and then was put on carbamezepine. She remained very much agitated for next 2 days, her blood pressure started to shoot up again, her urine output declined, serum urea and creatinine started to rise again. Intravenous methyl prednisolone pulse therapy was considered at that time, however she was noted to be tachypnic, distressed and her oxygen saturation dropped to $83 \%$. She was then urgently transferred to PICU, intubated and ventilated there. There was small amount of blood through ET tube. $\mathrm{Her} \mathrm{Hb}$ dropped to $6.0 \mathrm{~g} / \mathrm{l}$. Urgent $\mathrm{X}$ ray chest showed basal lung infiltrate on both sides.. Intravenous methyl prednisolone was started immediately at a dose of $30 \mathrm{mg} / \mathrm{kg}$ daily (for 3 days). PRBC was transfused. There was small amount of blood through ET tube on $2^{\text {nd }}$ day also but then stopped; she did not have any further bleeding per ET tube. On $5^{\text {th }}$ day (in PICU), she was extubated. She gradually tolerated oral feeds. She was then started on oral cyclophosphamide, total dose of $180 \mathrm{mg} / \mathrm{kg}$, to be taken over 90 days (approximately $2 \mathrm{mg} / \mathrm{kg} /$ day). Prednisolone was then given orally at a dose of $2 \mathrm{mg} / \mathrm{kg} /$ day. By that time, the official report of renal biopsy was available. It revealed diffuse mesangial and segmental endocapillary proliferation with karyorrhexis and necrosis. Segmental sclerosis was also seen. Few glomeruli showed fibro cellular crescent. EM showed mesangial immune complex deposits. Immunofluoroscence microscopy showed IgA, $\mathrm{C}_{3}$ and fibrinogen in the mesangium but no IgG, IgM or $\mathrm{C}_{1 \mathrm{q}}$. The features were compatible with HSP Nephritis Class III A (Focal endocapillary proliferation with crescent in few glomeruli).

Over the days she improved, her renal function returned to near normal. Almost a month later, she was discharged home on oral prednisolone $2 \mathrm{mg} / \mathrm{kg}$ every other day and oral cyclophosphamide for 3 months as prescribed before. Prednisolone was gradually tapered over approximately 9 months and then finally was taken off when her urine was completely \& persistently negative for protein and RBC. She continued to do well. Her blood pressure returned to normal and amlodipine was stopped. She was taken off Carbamezepine after 2 years. She is currently on no medication. Her physical and pubertal growth is optimum. She never had any recurrence of HSP during last 2 years of follow up with us. 


\section{Discussion}

Henoch Schönlein Purpura is a mild disease in children and usually self-limiting over few weeks. However complications can occur. Renal involvement in HSP is seen in $20-50 \%$ cases, however involvement varies from mild proteinuria, hematuria to severe nephritis, acute renal failure. Interstitial lung disease with slight radiological signs can also occur in many children during acute phase. Pulmonary hemorrhage is rare in prepubertal children but if it happens then it carries slightly better prognosis than in adults and adolescents. ${ }^{1-4}$ Other complications include GI bleeding and intracerebral hemorrhage; both these conditions add further to morbidity and mortality. ${ }^{5}$ Our patient had many grave complications of HSP, progressing over a short span of time. She seemed to have an unfavorable outcome. She had rapidly progressive glomerulonephritis, pulmonary hemorrhage, nephrotic syndrome, hypertension, intermittent intussusception, and cerebral vasulitis with seizure. Combination of such severe complications in one patient over a short span of time is very rare.

The optimum treatment for such cases remains unclear because of the lack of prospective randomized trials. Corticosteroid treatment alone does not seem to be effective in severe cases but a combination of pulse methylprednisolone and cyclophosphamide or azathioprine may be more beneficial. As of now, it is also not clear if iv pulse cyclophosphamide is better than oral cyclophosphamide. Our patient had combination of multiple severe complications however she was successfully treated with intravenous pulse methylprednisolone for three doses (followed by longterm oral prednisolone) and oral cyclophosphamide for 3 months. Her renal function recovered completely, other complications also resolved and she did not have any recurrence.

\section{Conclusion}

It is important to initiate very aggressive treatment in severe cases of Henoch Schönlein Purpura. In our experience, we found that combination of iv methylprednisolone pulse therapy followed by oral prednisolone plus oral cyclophosphamide to be very effective. However, other combinations like iv methylprednisolone plus iv monthly cyclophosphamide pulse or combination of corticosteroid and azathioprine or cyclosporine may also be equally effective.

\section{References}

1. Meadow SR. The prognosis of Henoch Schönlein Nephritis. Clin Nephrol 1978;9:87-90

2. Wright WK, Krous HF, Griswold WR, et al. Pulmanary vasculitis with hemorrhage in anaphylactoid purpura. Pediatr Pulmonol 1994;17: 269-271

3. Payton CD, Allison ME, Boulton- Jones JM. Henoch Schönlein Purpura presenting with pulmonary hemorrhage. Scott Med J 1987;32:26-27

4. Olson JC, Kelly KJ, Pan CG. Pulmonary disease and hemorrhage in Henoch Schönlein Purpura. Pediatrics 1992;89:1177-1181

5. Saulsbury FT. Henoch Schönlein Purpura in children. Report of 100 patients and review of the literature. Medicine (Baltimore) 1999;78:395-409. 